\title{
Based on the Genetic Algorithm to Optimize the QoS Routing Algorithm
}

\author{
Jing Yang \\ Dept. of Computer Science \\ Yunyang Teachers' College, Shiyan 442000, China \\ 80415@sohu.com
}

\begin{abstract}
This article introduce the genetic algorithm to solve the routing problem with QoS requirements, solve the traditional routing algorithms in completely the limitation of the NP problem. QoS routing optimization model is given, and the detailed design of genetic algorithm encoding scheme, fitness function, selection operator, crossover operator and mutation operator. Finally, the simulation experiment algorithm according to have better convergence and stability, in solving the problem of QoS routing is feasible.
\end{abstract}

Keywords: Cognitive Radio Networks, Channel Capacity Meeting Rate, Channel Stabilization, Genetic Algorithm, Routing Protocol

\section{Introduction}

Cognitive radio is a effective measure to solve the frequency spectrum is scarce, which are used by the cognitive user spectrum is obtained by means of perception, the authorized users, secondary utilization of unused spectrum to cognitive radio network routing presents new characteristics of contrary to the wireless network, such as diversity and time-varying spectrum set with nodes are available, and more dynamic network topology, only consider the hop routing may be because the link capacity and stability problem cause delayed routing big overhead. In this case, the cognitive radio network routing faces new problems and challenges, to have new requirements. Quality of Service (Quality of Service, QoS) has been a hotspot of research on network [1-3], in the network layer is the transmission must be satisfied by the Quality, meet two or more independent parameter constraint routing problem is a NP complete problem. In cognitive radio network, the node of the link between dynamic big lead to alternative routing performance gap between larger, for choosing a high performance routing "best" will the traditional network routing into optimization strategy of "go all out". Genetic algorithm is a global random search algorithm, the genetic algorithm is used to improve routing routing protocol will effectively solve the above two questions. Based on the above discussion, routing algorithms and protocols for cognitive radio is very necessary to research, this article routing problem focuses on cognitive users, due to its spectrum is discrete, the routing of cognitive network design problems, such as in addition to consider the hop to consider spectrum characteristics and stability of the link. This paper applies genetic algorithm to the routing, and then to improve the ondemand routing protocols make it suitable for cognitive radio network and routing strategy of this paper, based on more network topology information, choose the existence of the high performance routing. 
Routing technology is the key technology of the network layer and the network quality of service guarantees. User cognitive users in cognitive radio (CR) and authorized users (Primary radio user, PR user). CR users can use the authorization of free spectrum, but after the rise of PR users must exit this spectrum. Based on this mechanism, compared with the traditional network of fixed frequency, cognitive radio Ad Hoc network frequency change with time and space. Makes the routing algorithms and protocols for cognitive radio environment faces new problems. Is due to the dynamic spectrum, the connection between the nodes situation changes, the traditional network for cognitive radio network has the characteristics of high dynamic routing, if the routing for a link with the advent of PR user break probability is too large, will greatly reduce the performance of routing, this is cannot be ignored in the routing protocol, this is not involved in the traditional routing protocols. As far as possible in order to conform to the requirements of the business and even satisfy the QoS requirements of business, we must first consider the spectrum in the routing technology performance and the influence of the use right by, in addition to the traditional based on hop count, need to introduce a new measure to improve the cognitive performance of network routing. Routing algorithm is the soul of the routing protocol [4-6], the purpose is to find a best routing performance. Link between nodes in cognitive network quality change is big, lead to the source node to the destination node of the performance gap between alternative paths. In traditional routing protocol routing discovery mechanisms, ignores many potential routing, just do the routing performance of "the best", it is concluded that the routing is not necessarily the best. In order to satisfy the QoS requirements of the business route requests, as far as possible need right by the information processing and find out that exist in the network performance better path, so the routing algorithms and protocols have larger research space.

Routing algorithm is the core of the routing protocol, routing algorithms use protocols to collect the information such as the state of the network and the resources available to calculate the best route to the source node to destination node. The design of routing algorithm also affect the operation of the routing protocol, routing algorithm design goals usually include the following contents:

The optimal: what is the purpose of the routing algorithm is to find the optimal path. Optimal is only relative to one or more of the following measures of reasonable choice [7]. Simplicity: algorithm process should be as simple as possible, should be balance routing overhead and profit. Rapid convergence: because in the cognitive radio network topology structure because each node of the available spectrum varies, so the routing algorithm must be fast convergence. CR users fairness: routing algorithm should be equal. In recent years, with the amazing development of network technology, and constantly have new network business, Quality of Service (Quality of Service, QoS) has been a hotspot of research on network, because the QoS parameters with multiple constraints, general constraint conditions and the routing problem has proved to be npcomplete problem [8]. Genetic algorithm is a global random search algorithm, the researchers are widely used to solve np-complete problem, and a variety of combinatorial optimization problems.

QoS refers to the business need to transfer the requirements of the quality of service network of transmission parameters. The network layer QoS requirements can be described by a series of measurement parameters, such as transmission delay, delay jitter and network bandwidth and packet loss rate, etc. With the rapid development of wireless communication, more and more kinds network QoS business, more and more high to the requirement of network, different business for the choice of parameters is 
not the same, the network will provide different QoS for a growing number of business services. Business sends users will require network can ensure the transmission performance of necessary to communicate and quality of communication service to the network QoS demands can be reflected at the same time transmission delay, delay jitter and network bandwidth, describe the characteristics of parameters, such as packet loss rate ${ }^{[5]}$. In general, routing must satisfy the user's QoS parameters, to meet user demand for network QoS. This article will apply genetic algorithm to solve the problem of QoS routing, and an overview of the algorithm and the implementation process of the various steps.

\section{Related Works}

Genetic Algorithm (based Algorithm, GA) is based on the theory of natural selection and Genetic mechanism of the optimization method is developed, it simulates the biological evolution in nature, is a kind of efficient parallel global search method. It to the idea of "survival of the fittest" as the center, USES the evolution laws of nature, the out the individual difference, keep good individual genetic, it adaptively control the searching process to get the optimal solution, the algorithm of the whole operation process according to the evolutionary mechanism of the organism, starting from a random group, according to the situation of the environmental fitness "natural selection" excellent individuals to retain, and carries on the breeding, derived the next generation, a generation of inherited the good genes of the parent group, has a better performance, also may produce gene mutation in the breeding, genetic down from one generation to another. Genetic algorithm in computing the abstract process of organism evolution process, the problem of parameter space, replace with coding space, according to select individual fitness function, crossover and mutation operation for individuals in the group, resulting in a new species, forming an iterative process. Overall, the new generation of group received the evolution, constantly updated, continuous development, improve the ability to adapt to nature, to promote population evolution in the direction of optimization, and finally find out the optimal solution. Genetic algorithm can effectively solve belongs to np-complete problem, some scholars also apply it to the routing problem, QoS routing is np-complete problem, in some complicated cases it is difficult to find the optimal solution, seek out the satisfied solution in the routing is in conformity with the actual, and genetic algorithm is one of the best tools. Introducing genetic algorithm on-demand routing, routing for genetic operation when, in fact is a kind of network information mining processing, get better performance of the available potential routing.

\subsection{Genetic Algorithm Basic Steps}

First of all, we first introduce some basic concepts involved in genetic algorithm: group (population): also known as the population, generally consists of many individuals, it is a collection of several individuals. In each individual in the genetic algorithms are needed for a solution to solve the problem, so the group is actually a set of solutions. Chromosomes (chromosome): also known as individuals, each chromosome corresponds to a solution of the problem, after often encoded in genetic algorithm converts solution suitable string, according to the practical problems have different representations. Population size (population size): the required number of chromosomes in a population. Genes (gene): refers to an element of the chromosomes, the pieces of string, according to the practical problems and the ways of encoding genes 
can be a number, it may be a string of characters. According to the working process of genetic algorithm, the basic genetic operation steps for the following 3 steps:

\section{(1) The Genetic Code}

When solving specific problems, must be the target problem into the chromosome bit string of genetic algorithm, at the same time be able to chromosomes represented as the actual problem, namely to determine the encoding and decoding. To determine the optimization, GA individual phenotype set of space called a problem space. Genetic algorithm has strong robustness, general issues gene can be used in one dimension of fixed-length chromosome representation. Genetic encoding binary encoding, sequence code, tree coding, etc., is one of the most basic binary code.

\section{(2) Calculate the Fitness Function}

In order to implement the principle of survival of the fittest, must the adaptability evaluation of individual bit string. The fitness of an individual for the environment, is a kind of be used to measure the degree of individual fitness in a population parameter values, it is usually in the form of a fitness function value. So the individual fitness value of high and low, directly reflects the individual's survival ability strong and the weak in this environment. In general, if the chromosome bit string of genetic structure is good, then the individual will have higher adaptive function value, indicates that the individual can obtain higher value, have stronger ability to survive.

\section{(3) Genetic Operators}

In the genetic operations, the whole process is simulated in the process of natural selection and genetic mutation breeding, hybridization and wait for a phenomenon, so as to realize the evolutionary process of evolution. In general, the genetic operators are mainly three kinds of selection, crossover and mutation.

\section{1) Select Operator}

Choice, that is, from the current group choose the individuals with high fitness value to generate the mating pool [9]. It is "the fittest, survival of the fittest" simulation of natural evolution principle. Low fitness of individuals in the smaller probability genetic into the next generation of group, the high fitness individuals with larger probability genetic to the next generation. In fact is to determine in what way from the parent population from which the individual genetic group, a genetic algorithm to the next generation. At present there are mainly by adaptive value proportion, sorting, selection, league and elite selection, etc.

2) The Crossover Operator

In biological genetic and evolutionary process, mating restructuring is an important link in genes, determine the direction of evolution and features. Crossover operator of genetic algorithm (ga) is mimic natural genetic recombination process of sexual reproduction, according to a certain way for part two pairs of chromosomes swap genes, and thus form a new individual. Its role is to pass the original good genes to the next generation of individuals, and generate contains more complex genetic structure of the new individual. Groups within each individual random collocation in pairs, a pair of chromosomes in one or a few genes with a certain probability (crossover probability) exchanging position, generating new individuals. 


\section{3) Mutation Operator}

Mutation operator is used to simulate the nature evolution genetic algorithm of chromosomes in a certain gene mutations occur. In genetic algorithm, the mutation operation is based on a certain probability randomly take out from the group of a number of individuals, and then random selected individuals of a particular mutation. In order to guarantee the stability of population development, general mutation probability values is smaller. Introducing the mutation operator in genetic algorithm, on the one hand, can make to maintain the diversity of population in the process of evolution, to prevent "premature" phenomenon [10]; On the other hand can improve the local search ability of genetic algorithm.

\section{(4) Group Setting and Initialized}

Genetic algorithm (ga) was conducted on the solution group, and group set is fundamental for the performance of genetic algorithm. The diversity of the individuals in the population size, the larger the group, the more rich, the algorithm into the smaller the risk of local solution, but with the increase of population size, amount of calculation is also will increase significantly. If the population size is too small, can make the searching space of genetic algorithm is restricted, may produce "premature" phenomenon.

\section{(5) Control Parameters}

Genetic algorithms in the process of operation, has a set of parameters have significant effects on its operation performance, need reasonable choice and control, which makes the search of genetic algorithm to achieve the best performance. The operation of the need to choose parameters in genetic algorithm are mainly population size, crossover probability and mutation probability, etc.

\section{QoS Routing Algorithm based on Genetic Algorithm Design}

\subsection{Optimization Model to Describe}

In cognitive radio network QoS routing algorithm is described in the process of involves many variables, for the convenience of narrative, of these variables are defined as follows:

(1) cognitive Ad Hoc network can be represented as a directed graph $G=(V, E), V$ cognitive nodes of the collection, E said the collection of links between nodes.

(2) because each cognitive node that exist in the network of the maximum transmission power is limited, for $\mathrm{Pw}$, its biggest transmission range $\mathrm{R}$.

(3) the distance between the node $v_{i}$ and $v_{j}$ the node for $d_{i j}$, if $d_{i j}<R$, may be between nodes $v_{i}$ and $v_{j}$ the existence of a path. For a routing request, one of the most ideal state is one who can find a from the source node and destination node $\mathrm{d} s$ path between $\mathrm{p}$ (link) in e said path $\mathrm{p}$, not only has the minimum routing cost, but also at the same time satisfy the QoS requirements (three routing restrictions: here we assume that the time delay, delay jitter, packet loss rate), meet the requirement of biggest packet loss rate $L_{\max }$, and meet the requirement of the end-to-end delay $D_{\max }$, and satisfies the requirement of end-to-end delay jitter $\Delta_{\max }$. The most ideal state can be shown by the following optimization problem to describe. 
The listed optimization problem as follows:

$$
\min f
$$

The corresponding constraint conditions for:

$$
\begin{gathered}
L(p)=1-\prod_{e \in p}(1-L(e)) \leq L_{\max } \\
D(p)=\sum_{\mathrm{e} \in \mathrm{p}} D(p) \leq D_{\max } \\
\Delta(p)=\sum_{\mathrm{e} \in \mathrm{p}} \Delta(p) \leq \Delta_{\max }
\end{gathered}
$$

It is obvious that the QoS routing problem can be described as a constrained minimum optimization problem.

\subsection{Coding Scheme}

The coding scheme of genetic algorithm has a lot of, different coding can be selected according to different problems, common coding with binary coding, real number coding, etc. Nodes in the actual transmission distance is limited, we assume that the nodes within the transmission distance can communication. In order to reflect the real network topology, we use the graph adjacency matrix representation of the entire network routing tables, each node number, node adjacency matrix of row and column, there are links between two points, the corresponding element in the matrix is 1 , otherwise 0 . According to the network $G=(V, E)$, the routing table for the adjacency matrix A.

Tree coding is a kind of variable length coding mode, the said length is open, search solution, the growth of this coding way of chromosome can be free, the situation is consistent with the routing. Based on the thought of tree coding, by using a direct view of coding, paths of the 1-2-4-3-5 chromosomes is expressed as the length of each chromosome can range $\left(\begin{array}{llllll}1 & 2,2 & 4,4 & 3 & , 3 & 5\end{array}\right)^{\mathrm{T}}$ in length.

\subsection{Design of Fitness Function}

Fitness function is the only principle of evaluation of chromosome, it is a review of the individual, the larger the fitness, illustrate the performance of the path, the better, more can satisfy the QoS constraints, fitness function construction of the bridge between the optimization and genetic algorithm. Not only that, fitness function or subsequent choose copy operation standard, high fitness individuals have higher probability to survive in the select copy, reproduction, through genetic operators to make their offspring also has a good fitness. Low fitness of the individual is easier to be eliminated.

In this article, as set by the standards of the QoS routing fitness function is as follows:

$$
\begin{cases}0 & L(\mathrm{p})>L_{\text {max }} \vee D(p)>D_{\text {max }} \vee \Delta(p)>\Delta_{\text {max }} \\ 1 / Q_{p} & L(\mathrm{p}) \leq L_{\text {max }} \vee D(p) \leq D_{\text {max }} \vee \Delta(p) \leq \Delta_{\text {max }}\end{cases}
$$




\subsection{Selection Operator}

In genetic algorithm, the choice of replication operator reflects the evolution in the nature of the mechanism of "evolution". The design of selection operator has a crucial effect on the diversity of population. Diversity in the population difference would be prone to "premature" phenomenon of genetic algorithm, the algorithm stagnation. So rational selection operators can not only make maintain population diversity, still can make excellent individuals have survived. Simple genetic algorithm to choose way, generally using the roulette method, namely according to individual fitness and average fitness rate to determine the individual's choice. This method in the early some individuals have greater fitness, easy to replicate many of the individual to the next generation, it makes a single population, into local optimal solution, a "premature" phenomenon. In the later similar individual fitness, roulette couldn't reflect the excellent individual competitiveness, difficult to achieve superior bad discard. Therefore, this article USES the tournament and choosing elite reserved copy method, specific steps are as follows:

(1) the highest population fitness individuals directly elected, copied to the next generation of population.

(2) two individuals selected at random from the population, the probability of the same does not change according to the size of the fitness, compare two individual fitness value, high fitness individuals are copied to the next generation of population.

1) repeat step (2) $(\mathrm{K}-1)$ for population size $(\mathrm{K})$, the populations of the next generation of individuals, genetic operation to the next.

This has kept the best individual, avoid the tournament selection process of degradation, also let the population had better diversity, guarantee the convergence speed and avoid the local optimum.

\subsection{Crossover Operator}

Biological genetic recombination crossover operator is to simulate the nature of the process, in the genetic algorithm, crossover operator is the main way to produce new individual, has the vital role that determines the global search ability of the algorithm, if the crossover probability and the $\mathrm{cp}$ is too small, each generation of new individuals, most individuals are copied to the next generation, easy to slow convergence stagnation, $\mathrm{cp}$ and probability is bigger, may damage more excellent individuals, are ultimately to random search algorithm, goes against the original intention of the genetic algorithm.

In this paper, by adopting the idea of dynamic crossover probability, in the process of algorithm, the timely change of $\mathrm{cp}$ value, genetic algorithm convergence speed and ease the contradiction between the two species diversity. Expression is as follows:

$$
p_{c}=p_{c i}-\left(p_{c i}-p_{c \min }\right) d / D
$$

Where $\mathrm{d}$ in type for the current evolution algebra; $\mathrm{D}$ is the overall evolution algebra; As the initial crossover probability, as minimal crossover probability.

Adopted crossover probability in (6), early on, the crossover probability is bigger, can avoid the species diversity of falling rapidly, so as to produce many new individual. In late evolution, high fitness of chromosomes in a population is more, the population will collective to be near the optimal solution, the crossover probability of small to ensure the fitness of individuals in the population proportion, increase the convergence speed.

Algorithm of cross produce offspring chromosomes specific steps are as follows 


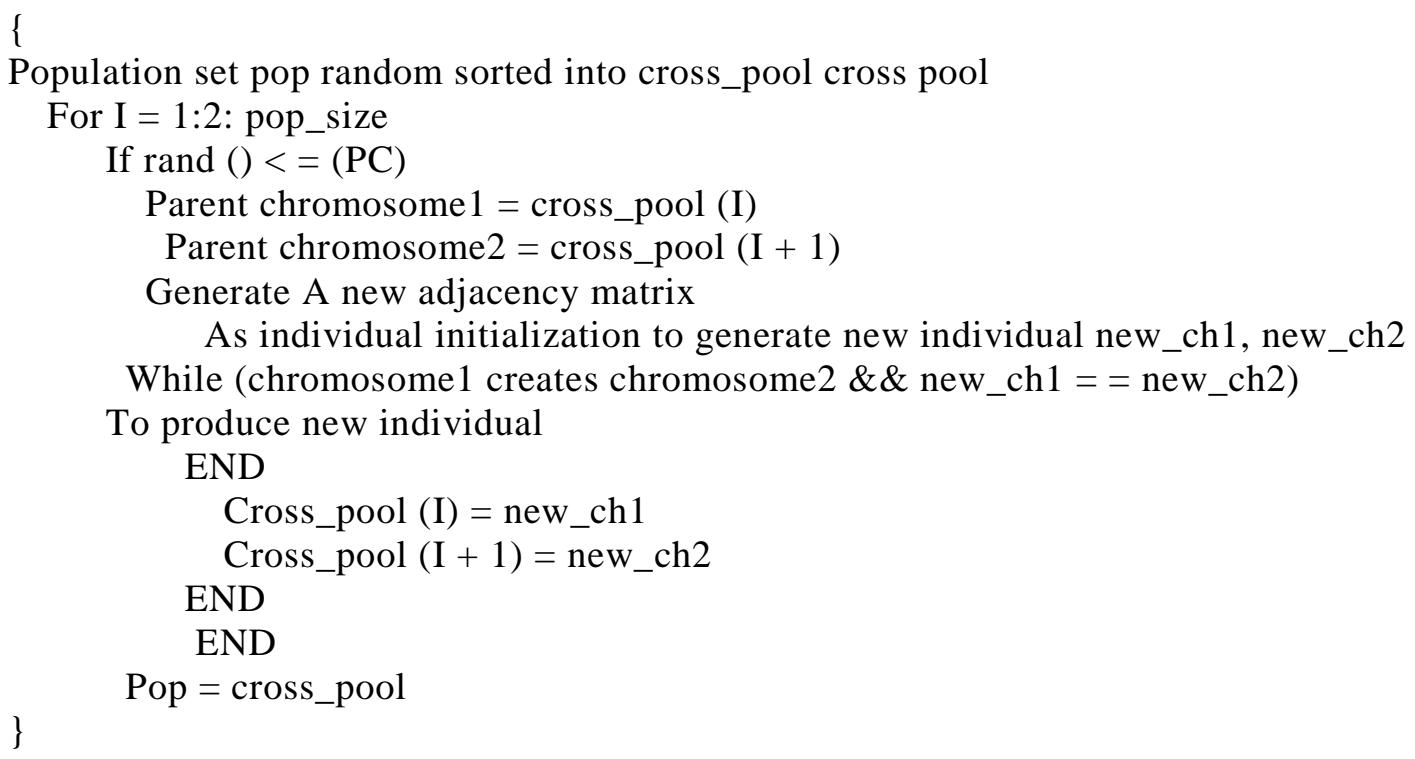

\section{Simulation Results and Analysis}

\subsection{Network Topology and Parameters Setting}

In order to examine the actual performance of the new algorithm, design the following simulation experiment. First routing algorithm simulation is based on the network topology, in order to make the network topology has universality, neither to use a specific network topology, also did not use a completely random generation network topology. Because in random network topology node density and spacing is difficult to control, leading to excess long island node and link, the network to test the algorithm will affect the reliability of the results. In order to make the applied to the simulation of the network topology and the actual network closer, we adopted k-means clustering network topology model.

This all nodes according to their location model is divided into several classes, calculate an average in each class is the "center", all center of object is the topology of a node. In this experiment to produce 100 nodes in the network topology, randomly generated in the coordinates are now 2000 nodes, then according to the $\mathrm{K}$ - means algorithm to calculate the 100 class, the class average node is the last of the topology of 100 nodes. K-means clustering network topology model generated 100 nodes.

Edge set is generated according to the above hypothesis, when the distance of any two nodes is less than the transmission distance, we think that they can connect. Genetic algorithm of control parameters on the algorithm performance has a great influence, according to the routing of the specific conditions of the model and the algorithm of genetic algorithm and the experimental parameters are set as follows: group size choose between 20 and 50, according to the actual circumstances of the crossover and mutation operator is designed in this paper, the initial mutation probability, mutation probability, the minimum initial crossover probability, crossover probability, the smallest end algebra choice between 50 to 100. Link delay between [10 $\mathrm{ms}-30 \mathrm{~ms}]$ uniform distribution, the maximum time delay constraint on $3 \mathrm{dmax}=240$ $\mathrm{ms}$, link packet loss rate in $\left[\begin{array}{ll}0.01 & 0.1\end{array}\right]$ between uniform distribution, the biggest packet loss rate constraint $\operatorname{Lmax}=0.08$, delay jitter between $[0-5 \mathrm{~ms} \mathrm{~ms}]$ uniform distribution, 
the most large delay jitter Max $=40 \mathrm{~ms}$, cost matrix evenly distributed between $[0.01$ 0.05]. We select the source node to 5, the destination node routing experiment for 100 .

Application of genetic algorithm is designed for simulation experiment, the experiment mainly verify the convergence of the algorithm, the convergence speed and stability, etc. Experiments on the algorithms in this paper the highest fitness and the average fitness of each iteration to draw the following Figure 1.

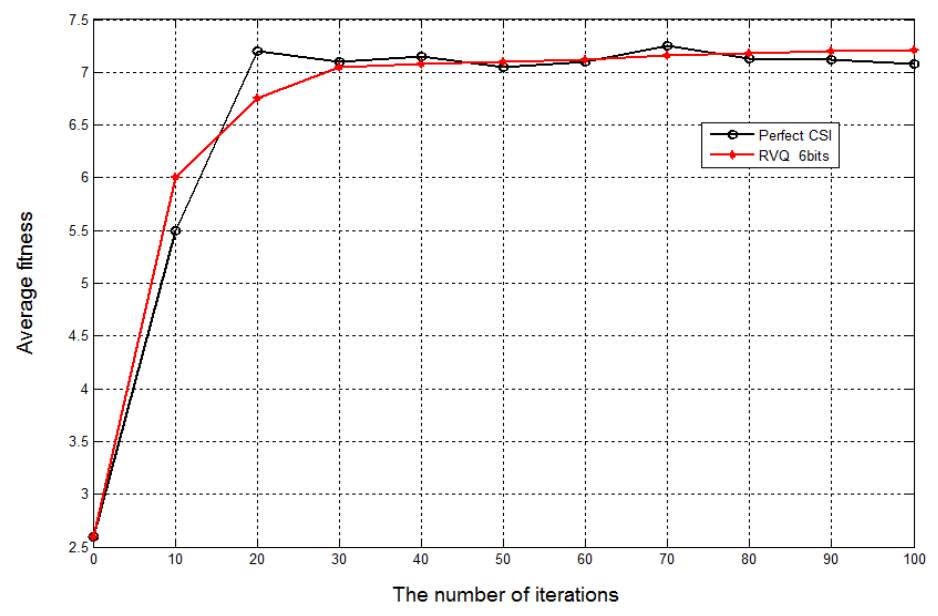

Figure 1. The Average Fitness VS Number of Iterations

Figure 1 to test the algorithm of population average fitness as the changes of the number of iterations increases. Network scale 100 nodes in the simulation experiment, the topology is shown in Figure 4.8, the population size of 50, and the other parameters are described as above said. Can be seen from the diagram, the SGA as crossover and mutation parameters constant, the average fitness is beginning to rise faster, but with the increase of algebra, the average fitness increase speed significantly slower. In this paper, the early stage of the algorithm with adaptive crossover mutation probability as far as possible let the population diversity, so the average fitness up relatively slow, late average fitness is stable. By experiment result algorithm has fast convergence in the trend of the optimal solution, so it is concluded that the algorithm has good convergence, and meet the requirements of routing algorithm for fast convergence.

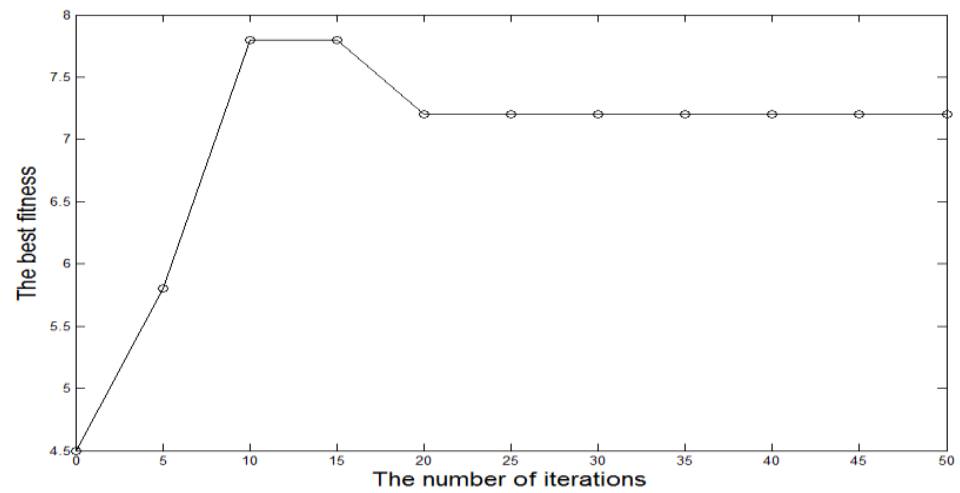

Figure 2. The Best Fitness and the Relationship between the Number of Iterations 
Figure 2 for test algorithm is optimal in the changes of the fitness with the increase of the number of iterations. Experiments for 100 nodes network size, the topology is shown in figure, the population size of 50, other parameters given by the above. Can see algorithm with the increase of the number of iterations, the best fitness is to approach the optimal solution, and the optimization speed is faster. Accord with the actual routing problem in the path selection of real-time characteristics. Can quickly find the optimal solution to get the fastest.

Beautiful path, avoid topology changes in routing failure. One aspect of the stability of the algorithm is also tested, stable and fast to find the optimal solution of the more important, in the practical routing node number 100 in the experiment, the topology is shown in figure 4.8, under different population size and number of iterations, the algorithm for the simulation experiment of repeated 50 times, 50 times is obtained by statistical experiment, the optimal solution of the mean and the probability of the optimal solution are obtained.

\section{Conclusion}

This article introduce the genetic algorithm to solve the routing problem with QoS requirements, solve the traditional routing algorithms in completely the limitation of the NP problem. QoS routing optimization model is given, and then detailed introduces the encoding scheme, fitness function of genetic algorithm, the design of selection operator, crossover operator and mutation operator. Finally, the simulation experiment algorithm according to have better convergence and stability, in solving the problem of QoS routing is feasible.

\section{References}

[1] P. Kolodzy, "Spectrum policy task force", Fed. Commun. Comm., Washington, DC, Tech. Rep. ET Docket, no. 02135, (2002).

[2] I. F. Akyildiz and W. Y. Lee, "Next generation/dynamic spectrum access/cognitive radio wireless networks: a survey", Computer Networks, vol. 50, no. 13, (2006), pp. 2127-2159.

[3] M. McHenry, "Report on spectrum occupancy measurements", shared Spectrum Company, (2005).

[4] M. Dillinger and K. Madani, "Software Defined radio: Architectures, systems, and functions", John Wiley \& Sons Inc, (2003).

[5] E. R. Kwerel and J. Williams, "A proposal for a rapid transition to market allocation of spectrum", Federal Communications Commission, Office of Plans and Policy, (2002).

[6] G. R. Faulhaber and D. Farber, "Spectrum management: Property rights, markets, and the commons", Rethinking rights and regulations: institutional responses to new communication technologies, (2003), pp. 193-226.

[7] J. Mitola III and G. Q. Maguire Jr., "Cognitive radio: making software radios more personal", Personal Communications, IEEE, vol. 6, no. 4, (1999), pp. 13-18.

[8] J. Mitola, "Cognitive radio: An integrated agent architecture for software defined radio", Doctor of Technology, Royal Inst. Technol.(KTH), Stockholm, Sweden, (2000), pp. 271-350.

[9] F. C. Commission, "Report and Order (FCC 05-57): Facilitating Opportunities for Flexible, Efficient, and Reliable Spectrum Use Employing Cognitive Radio Technologies”, Etdocket, no. 03-108, (2005).

[10] Z. Weizhe, H. Mingzeng, Z. Hongli and L. Kaipeng, "A Multi objective Evolutionary Algorithm for Grid Job Scheduling of Multi-QoS Constraints”, Journal of Computer Research and Development, vol. 11.

\section{Authors}

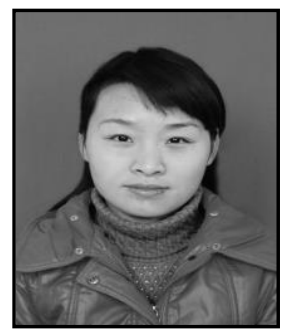

Jing Yang, received her Bachelor of Science degree in Computer Science and Technology from Hubei Normal University, CHINA in 2003. She is currently working toward her Master's Degree of Science in computer applications. She is mainly researching on routing algorithm based on network optimization, data mining, and Internet of Things. 